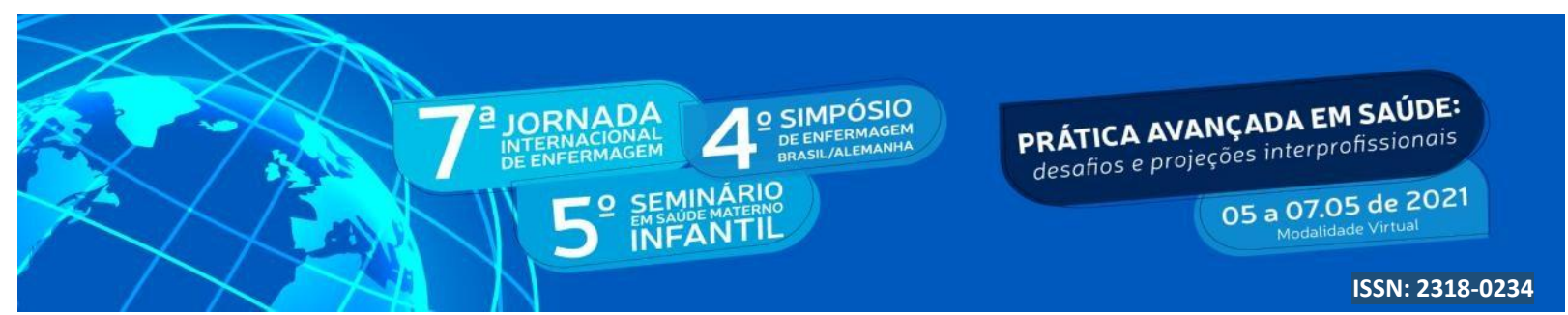

DOI: http://doi.org/10.48195/jie2021-137

\title{
A UTILIZAÇÃO DAS PRÁTICAS INTEGRATIVAS E COMPLEMENTARES EM SAÚDE EM PACIENTES ONCOLÓGICOS: REVISÃO DE LITERATURA
}

\section{Aliny da Silva dos Santosi ; Flávia Camef Dorneles Lenz ${ }^{2}$; Nathália Fortes Schlotfeld ${ }^{3}$; Letícia Martins Machado ${ }^{4}$}

\section{RESUMO}

Objetivo: identificar a aplicação das Práticas Integrativas e Complementares em Saúde no cuidado em Oncologia. Metodologia: trata-se de uma revisão narrativa de literatura, na qual a busca pelos artigos foi realizada na base de dados Literatura Latino-Americana e do Caribe em Ciências da Saúde (Lilacs) utilizando-se os descritores "Terapias Complementares" AND "Oncologia" no mês de março de 2021. Resultados: os dois artigos que compuseram esta revisão são dos anos 2008 e 2015, sendo ambos realizados no Sul do Brasil e publicados na área da enfermagem e resultantes de pesquisas qualitativas e quantitativas ambos no idioma português. Conclusão: $\mathrm{O}$ estudo mostrou diversos resultados positivos em relação a melhora do estado físico e mental após a aplicação destas práticas.

Palavras-chave: Atenção integral à saúde; Enfermagem; Oncologia; Terapias Complementares.

\footnotetext{
ABSTRACT

Objective: to identify the application of Integrative and Complementary Health Practices in Oncology care. Methodology: this is a narrative literature review, in which the search for

${ }^{1}$ Autora. Acadêmica de Enfermagem. Universidade Regional Integrada do Alto Uruguai e das Missões, campus de Santiago. E-mail: alinydasilvas@gmail.com

${ }^{2}$ Coautora. Enfermeira. Graduada pela Universidade Regional e Integrada do Alto Uruguai e das Missões, campus de Santiago. E-mail: flaviacamefd@gmail.com

${ }^{3}$ Coautora. Acadêmica de Enfermagem. Universidade Regional Integrada do Alto Uruguai e das Missões, campus de Santiago. E-mail: nathaliafschlotfeldt@gmail.com

${ }^{4}$ Orientador. Mestre em Enfermagem. Universidade Regional Integrada do Alto Uruguai e das Missões, campus de Santiago. E-mail: lehmachado@yahoo.com.br
} 


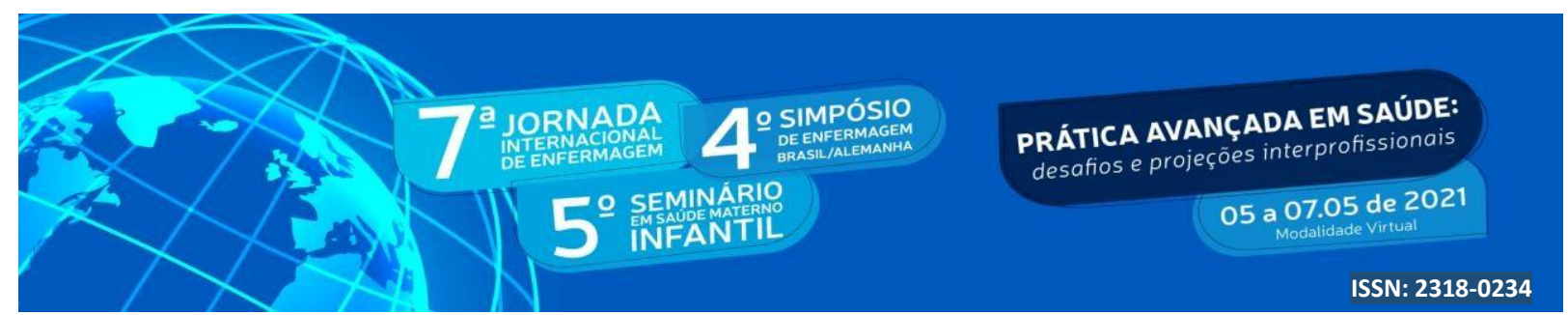

articles was carried out in the Latin American and Caribbean Literature in Health Sciences (Lilacs) database using the descriptors "Complementary Therapies" AND "Oncology" In March 2021. Results: the two articles that made up this review are from the years 2008 and 2015, both being carried out in the South of Brazil and published in the field of nursing and resulting from qualitative and quantitative research both in the Portuguese language. Conclusion: The study showed several positive results regarding the improvement of the physical and mental state after the application of these practices.

Key Words: Complementary Therapies; Oncology; Comprehensive health care; Nursing.

\section{INTRODUÇÃO}

Sendo a segunda causa de mortalidade no Brasil e no mundo, o câncer além de ter um grande significado epidemiológico, apresenta diversas repercussões nas questões sociais. Esse aumento tem se refletido na elevação do número de tratamentos ambulatoriais, das taxas de internações hospitalares e dos recursos públicos demandados para custear os tratamentos. A incidência das neoplasias malignas tem uma distribuição diversificada entre as regiões do país e necessita de diversos tipos de ações e serviços de saúde (LINS; SOUZA, 2018).

O câncer traz consigo diversas incertezas no que tange a sua evolução e aos resultados do tratamento, causando angústia, sofrimento, insegurança, dor e muitas vezes revolta nos pacientes e seus familiares. Esses sentimentos desencadeados podem ser atribuídos à compreensão, trazida pelo senso comum, de que se trata de uma doença sem perspectiva de cura, e muitas vezes tendo como prognóstico somente a morte (CARMO et al., 2019). Diante disso, é necessário que o paciente desenvolva ferramentas de enfrentamento, mudanças comportamentais e cognitivas, adaptações individuais e coletivas para o processo de adoecimento (SILVA et al., 2020).

Assim, ao cuidar do paciente oncológico, os enfermeiros lidam com alto grau de comprometimento emocional, uma vez que, cuidam de pacientes em tratamentos prolongados 


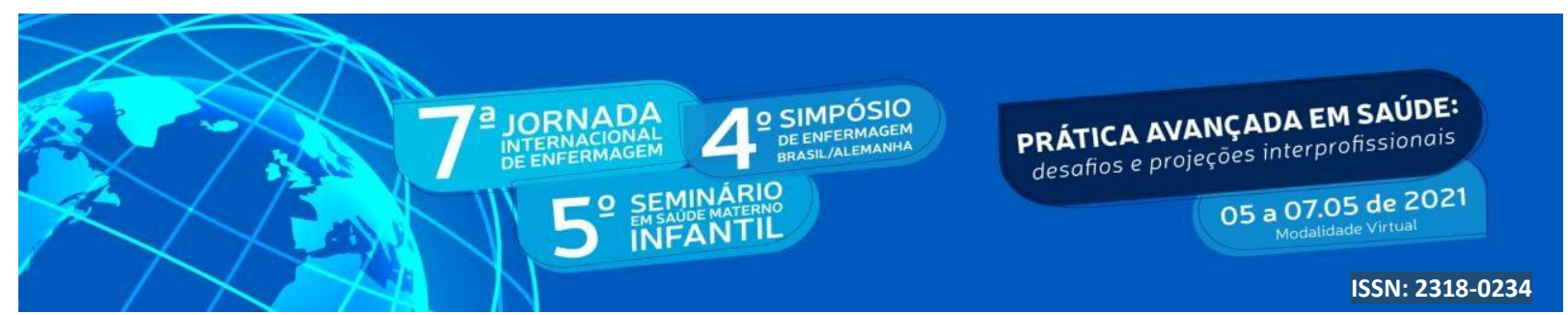

que, muitas vezes, resultam em diversos efeitos colaterais e dificuldades, como alterações no cotidiano, na autoestima e na autoimagem, além de vivenciarem experiências com a finitude da vida, gerando grande impacto na sua identidade pessoal e profissional e de toda equipe envolvida no cuidado (CARMO et al., 2019).

Nesse sentido, as Práticas Integrativas e Complementares em Saúde (PICS) surgem como possibilidade de favorecer o processo de cuidado. Elas são abordagens de cuidado e de recursos terapêuticos, que procuram estimular os mecanismos naturais de prevenção de agravos e de recuperação da saúde por meio de tecnologias eficazes, seguras e menos invasivas com destaque na escuta acolhedora, na criação do vínculo terapêutico e na aproximação do ser humano com o meio ambiente e a sociedade. No Brasil, a legitimação e a institucionalização dessas abordagens de atenção à saúde teve início com a criação da Política Nacional de Práticas Integrativas e Complementares (PNPIC), em 2006 (BRASIL, 2015).

No ano de 2018, por meio da Portaria $\mathrm{n}^{\mathrm{o}}$ 702, foram instituídas 29 práticas integrativas que compõe o quadro de oferta, sendo elas: Homeopatia, Medicina Tradicional Chinesa/Acupuntura, Ayurveda, Naturopatia, Plantas Medicinais e Fitoterapia, Termalismo Social/Crenoterapia, Reiki, Yoga, Medicina Antroposófica, Arteterapia, Biodança, Dança Circular, Meditação, Musicoterapia, Reflexoterapia, Osteopatia, Quiropraxia, Shantala, Terapia Comunitária Integrativa, Apiterapia, Aromaterapia, Bioenergética, Constelação Familiar, Cromoterapia, Geoterapia, Hipnoterapia, Imposição de mãos, Ozonioterapia e Terapia de Florais (BRASIL, 2018).

\section{OBJETIVO}

Estre trabalho tem como objetivo identificar a aplicação das Práticas Integrativas e Complementares em Saúde no cuidado em Oncologia. 


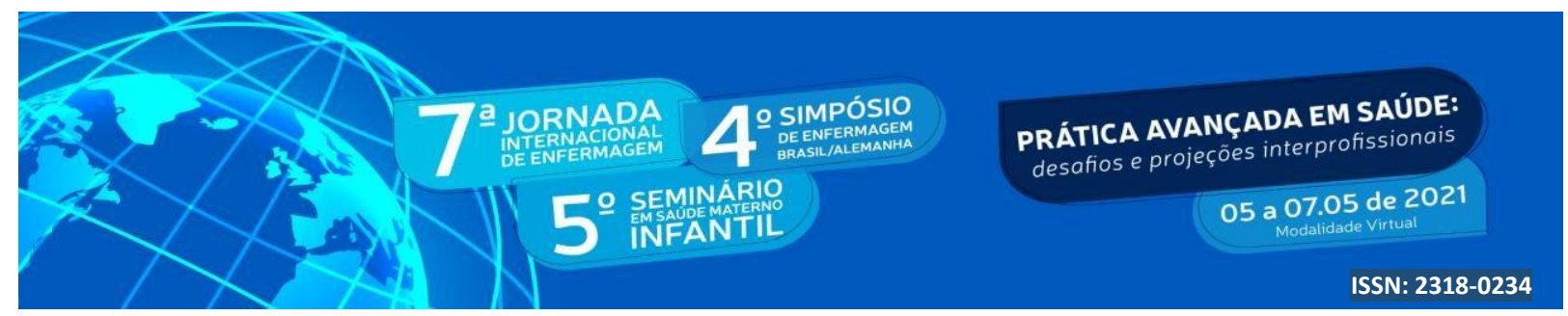

\section{METODOLOGIA}

Trata-se de um estudo de Revisão Narrativa de Literatura (RNL), no qual a busca pelos artigos foi realizada na base de dados Literatura Latino-Americana e do Caribe em Ciências da Saúde (Lilacs) utilizando-se os descritores "Terapias Complementares" "Oncologia", associados com operador boleano "AND”.

Para este levantamento bibliográfico foram consultadas, no mês de março de 2021, a partir da leitura dos artigos, norteada pela seguinte questão: O que tem sido produzido acerca da aplicação das Práticas Integrativas e Complementares em Saúde aplicadas no cuidado em Oncologia? O recorte do período de publicação dos artigos foi de 2006 a 2021, com objetivo de selecionar estudos realizados após a publicação da Política Nacional de Práticas Integrativas e Complementares em Saúde (PNPICS).

A busca inicial foi composta por 11 produções. Os critérios de inclusão foram: artigos de pesquisa disponíveis na íntegra, online e gratuitamente, nos idiomas português, inglês ou espanhol.

Foram excluídos os estudos que não abordassem a temática. Os artigos duplicados foram contados somente uma vez. Para o acesso ao texto completo, foram usados os seguintes recursos: link disponível diretamente na própria base de dados selecionada, e busca no portal do periódico em que o artigo foi publicado.

Por fim, a partir do estabelecimento dos critérios de inclusão e exclusão, o corpus desta revisão constituiu-se em dois artigos que eram de interesse do objetivo do estudo proposto.

\section{RESULTADOS E DISCUSSÃO}




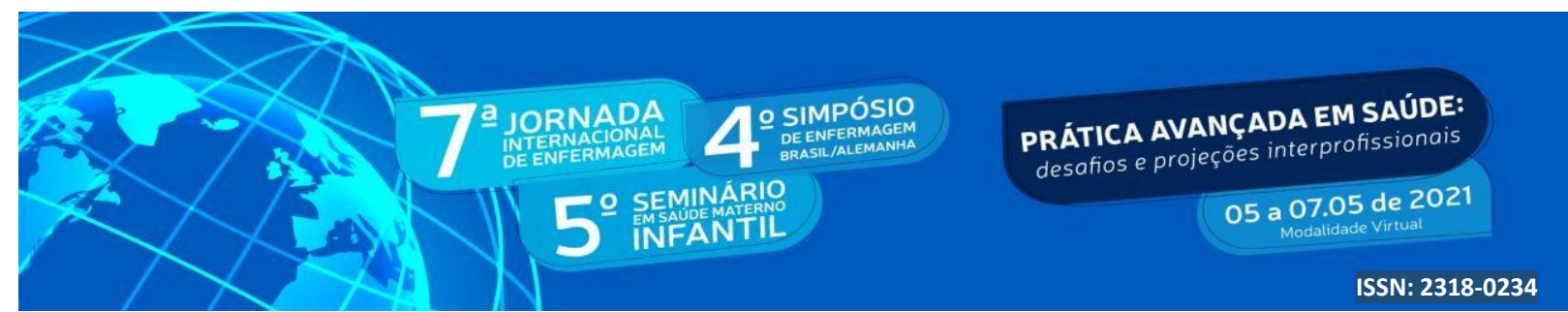

Quanto a caracterização dos estudos, os dois artigos que compuseram esta revisão são dos anos, 2008 e 2015. Sendo ambos realizados no Sul do Brasil e publicados na área da enfermagem. Resultantes de pesquisas qualitativas e quantitativas ambos no idioma português. A partir do momento em que a pessoa tem o diagnóstico de câncer e passa a realizar tratamento quimioterápico, muitos sentimentos negativos são associados a esta experiência e alguns autores consideram "que seu estigma esteja além do ser temido pela representação social de morte, sofrimento, etc.", denominando o câncer como doença transformadora, sendo que estas mudanças nem sempre são perpassadas por momentos felizes (SALIK, 2013).

Em sua terapêutica, o paciente oncológico anseia um enfoque integral, assim compreende-se a utilização das terapias complementares como um recurso positivo, pois a literatura aponta que há significativa melhora na qualidade de vida e diminuição do estresse causado pela doença e tratamento (BRASIL, 2012). Tais recursos envolvem abordagens que buscam estimular os mecanismos naturais de prevenção de agravos e recuperação da saúde por meio de tecnologias eficazes e seguras, com ênfase no desenvolvimento do vínculo terapêutico, escuta acolhedora e integração do ser humano com o ambiente e a sociedade (SPADACIO; BARROS, 2008).

A Oncologia Integrativa é um ramo da Medicina Integrativa que contempla a integração das práticas alternativas e complementares aos tratamentos realizados para o câncer como a quimioterapia, radioterapia, cirurgia e terapia molecular. Esta forma de cuidado busca constantemente o protagonismo do paciente oncológico em seus processos assistenciais, bem como resgatar os princípios da bioética estimulando a autonomia do ser cuidado (SIEGEL; BARROS, 2013).

Em um dos estudos que emergiram nesta revisão, teve como objetivo conhecer a prevalência da utilização das terapias alternativas por pacientes em tratamento quimioterápico. Por meio de uma pesquisa quantitativa com a participação de 65 pacientes, apresentou como 


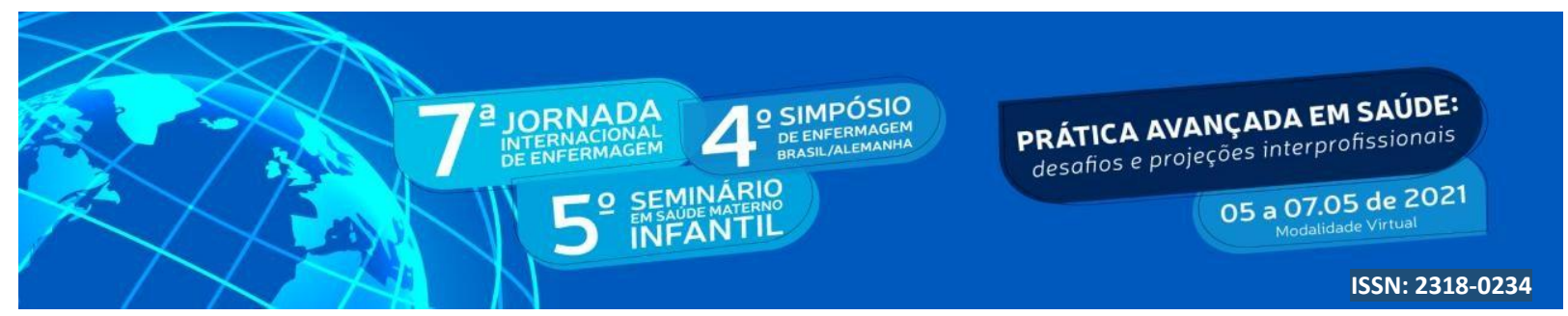

resultado, a utilização das PICS por $69 \%$ dos pacientes, sendo a fitoterapia com maior predominância (JACONDINO; AMESTOY; THOFEHRN, 2008).

O termo fitoterapia designa a profilaxia e também o tratamento de condições de saúde através das plantas e por partes delas, tais como: folhas, flores, raízes, frutos ou sementes e pelos seus preparados (FINTELMANN; WEISS, 2010). É necessário que haja a comprovação da sua eficácia e dos riscos potenciais que podem oferecer, sendo que há uma legislação que norteia os registros dos medicamentos fitoterápicos junto à ANVISA (Agência Nacional de Vigilância Sanitária) (MOURA; et.al., 2014).

A fitoterapia e o uso de plantas medicinais também emergiram em outro estudo realizado no sul do Brasil com objetivo de conhecer as terapias integrativas e complementares utilizadas pelos pacientes em quimioterapia oncológica, por meio de uma pesquisa qualitativa realizada com seis pacientes (LIMA, et al., 2015)

As plantas medicinais compreendem uma forma de cuidar empírica, oriunda do saber popular construído ao longo do tempo, conformando-se em uma incorporação cultural ao cuidado oficial em saúde, baseada em uma visão integral a sua ação terapêutica faz parte do ambiente no qual o ser humano está inserido (FEITOSA; et.al, 2015).

Além disso, o estudo apontou ainda o uso da homeopatia pelos pacientes oncológicos. A homeopatia é uma técnica milenar da Medicina Holística que usa substâncias altamente diluídas para alívio de sintomas, no contexto da Oncologia Integrativa, pode ser usado nos estágios terminais, aliviando a dor. Estudos mostram que pode haver diminuição dos níveis de cortisol, frequência cardíaca e pressão arterial e aumento da atividade das células Natural Killer nos indivíduos expostos à natureza em relação ao ambiente urbano (FULOP, et.al., 2017).

Outros estudos também apontam que por meio do yoga há uma melhora do estresse, ansiedade, depressão e qualidade de vida, fadiga, insônia e condicionamento físico. Yoga é uma prática antiga que incorpora todo o corpo e inclui trabalhar com movimentos e posições (asanas), meditação e exercícios de respiração (pranayama), que têm como objetivo criar uma harmonia entre corpo e mente e permitir uma profunda conexão consigo mesmo, estimulando 


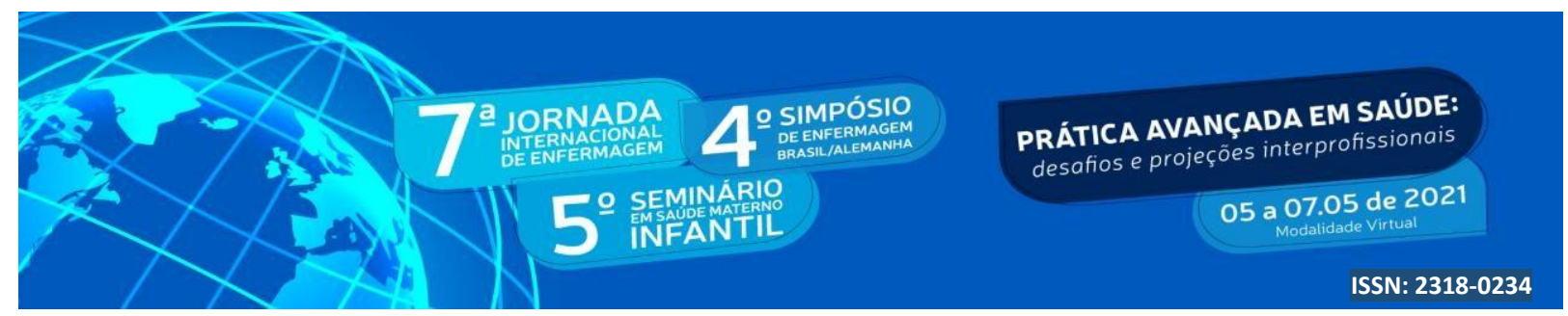

o sistema nervoso, relaxando a mente e o corpo e melhorando a flexibilidade (CARLSON; et.al., 2017).

O estudo observacional liderado por Lopez et.al (2018), cita melhora clínica e estatística dos sintomas de fadiga, falta de ar, ansiedade e do bem-estar geral, após sessão de meditação. Podendo ser descrita como um estado fisiológico holometabólico, na meditação o praticante permanece relaxado, porém alerta e focado, por meio de técnicas que incorporam visualização, respiração, com movimentos ou não, e podem ser praticadas sozinhas ou em grupo.

A prática mais estudada da Medicina Tradicional Chinesa atualmente é a acupuntura, definida como a inserção de agulhas finas e pequenas em pontos específicos do corpo, para tratar ou prevenir sintomas. Um estudo realizado na Universidade do Texas, com pacientes oncológicos internados que estavam recebendo acupuntura entre dezembro de 2014 e dezembro de 2015, relata que após a primeira sessão 74\% dos pacientes relataram melhora da dor. Foi reportada também melhora na náusea, ansiedade, sonolência e fadiga e para os que realizaram mais de uma sessão, evidenciou-se também alívio em distúrbios do sono, ansiedade, dor e sonolência (GARCIA, et.al., 2018).

A adaptação da massagem tradicional para o paciente com câncer, criando assim a Massagem Oncológica, levou em consideração precauções especiais como história de tratamento, cirurgia recente, medicações em uso, presença de doença metastática e exames laboratoriais. A técnica inclui mudanças na pressão aplicada durante a sessão e restrição de algumas partes do corpo (LOPEZ; MAO; COHEN, 2017). Estudos mostram que a massagem pode ser efetiva na melhora do humor e diminuição de ansiedade, depressão, náusea e dor (LOPEZ, et.al, 2017).

O Reiki é uma terapia holística baseada no conceito de energia vital (Rei significa universal e $\mathrm{Ki}$, força vital). Idealizada por Mikao Usui no começo do século 20, consiste em estimular os canais de energia do paciente por meio da colocação das mãos do praticante em diversas posições sobre ou levemente acima do corpo do paciente (QUEST; ROBERTS, 2011). 


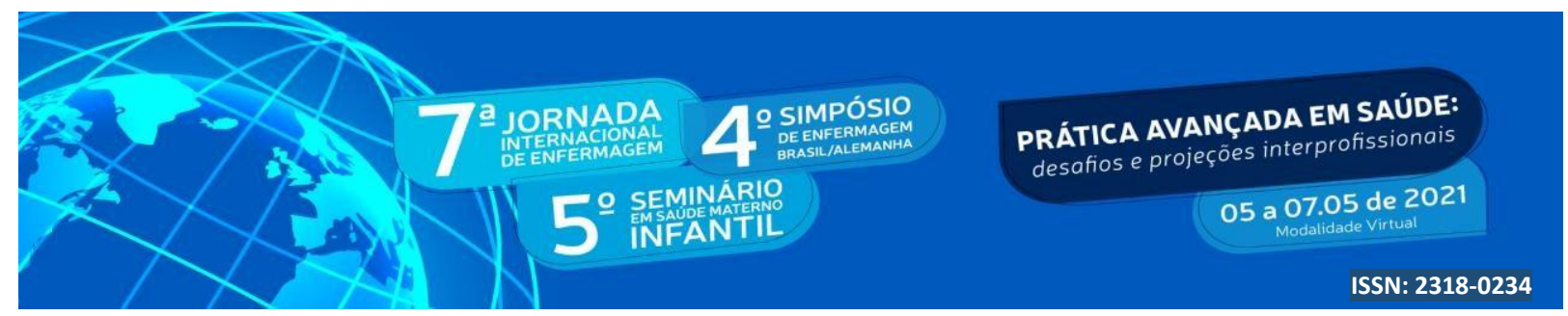

Os pacientes que receberam o tratamento com o Reiki relataram melhora no bem-estar geral e de alguns sintomas, mas nem todos os ensaios clínicos apresentaram resultados significativos. A melhora de sinais e sintomas como dor, fadiga, estresse, ansiedade sugerem que esta prática pode ser introduzida na prática assistencial ou recomendada a pacientes submetidos a quimioterapia ambulatorial (BEULKE, 2019).

Outro resultado importante relacionado à inclusão das práticas de medicina integrativa pelos pacientes oncológicos está relacionado à diminuição da necessidade do uso de medicações sintomáticas, como analgésicos opioides, analgésicos não opioides, antiheméticos, antidiarreicos, laxantes e ansiolíticos (SHALOM, et.al, 2017).

\section{CONCLUSÃO}

$\mathrm{O}$ estudo mostrou que durante o tratamento, os pacientes oncológicos sofrem com diversos efeitos adversos e diante disso surge a necessidade da utilização de terapias complementares. A revisão dos artigos apresentou diversos resultados positivos em relação a melhora do estado físico e mental após a aplicação destas práticas.

\section{REFERÊNCIAS}

BEULKE, S. L. et al. Reiki no alívio de sinais e sintomas biopsicoemocionais relacionados à quimioterapia. Cogitare enferm. Disponível em: http://dx.doi.org/10.5380/ce.v24i0.56694 Acesso em: 16 março 2021.

BRASIL. Ministério da Saúde. Gabinete do Ministro. Portaria n ${ }^{\circ}$ 971, de 03 de maio de 2006.

BRASIL. Ministério da Saúde. Portaria nº 702, de 21 de março de 2018. 


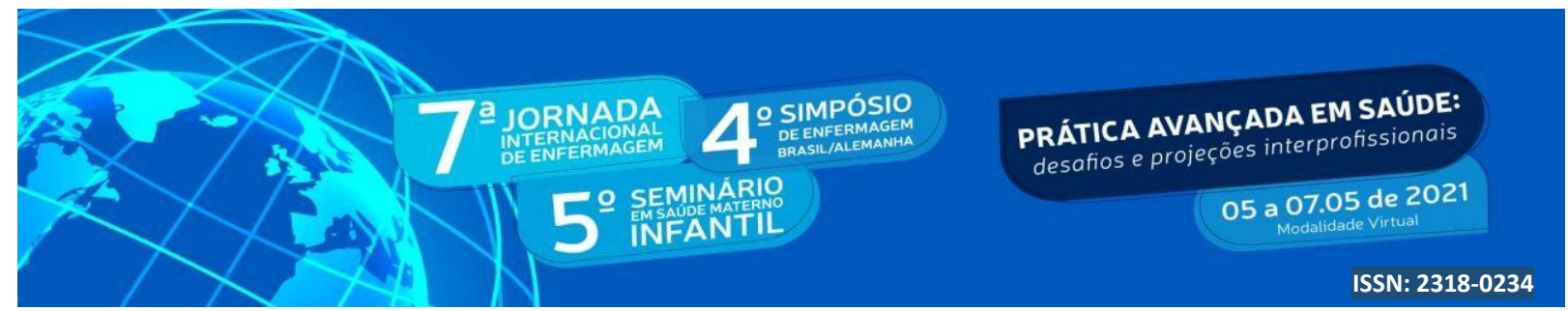

BRASIL. Ministério da Saúde. Secretaria de Atenção à Saúde. Departamento de Atenção Básica. Práticas integrativas e complementares: plantas medicinais e fitoterapia na Atenção Básica. Brasília, p. 156, 2012.

CARLSON, L. E., et al. Mind-Body Therapies in Cancer: What Is the Latest Evidence? Curr Oncol Rep. Current Oncology Reports, v. 19, n. 10, 2017.

CARMO, R. A. L. O. et al. Cuidar em Oncologia: Desafios e Superações Cotidianas Vivenciados por Enfermeiros. Revista Brasileira de Cancerologia, v. 65, n. 3, p. 1-10, 2019. FEITOSA, M.H., et.al. Plantas medicinais como recurso terapêutico entre funcionários do Centro de Ciências Biológicas e da Saúde da Unimontes. Revista Unimontes Científica, v.17, n. 1, p. 50-59, 2015.

FINTELMANN, V.; WEISS, R.F. Manual de fitoterapia.11 a ed. Rio de Janeiro: Guanabara Koogen; 2010.

FULOP, J.A.; GRIMONE, A.; VICTORSON, D. Restoring Balance for People with Cancer Through Integrative Oncology. Prim Care - Clin Off Pract. v. 44, n. 2, p. 323-35, 2017.

GARCIA, M. K.; COHEN, L.; SPANO, M.; SPELMAN, A.; HASHMI, Y.; CHAOUL, A., et al. Inpatient Acupuncture at a Major Cancer Center. Integr Cancer Ther. v. 17, n. 1, p.148 52, 2018.

JACONODINO, C. B.; AMESTOY, S. C.; THOFEHRN, M. B. A utilização de terapias alternativas por pacientes em tratamento Quimioterápico. Cogitare Enferm, v. 13, n. 1, p. 6166. Jan/Mar 2018.

LINS, F. G; SOUZA, S. R. Formação dos enfermeiros para o cuidado em oncologia. Rev enferm UFPE v. 12, n.1, p.66-74, Recife, 2018.

LOPEZ, G. et al. A Pragmatic Evaluation of Symptom Distress After Group Meditation for Cancer Patients and Caregivers: A Preliminary Report. J Pain Symptom Manage [Internet]. Elsevier Inc; v. 55, n. 5, p. 1321-1326, 2018.

LOPEZ, G. et al. The effects of oncology massage on symptom self-report for cancer patients and their caregivers. Support Care Cancer. Supportive Care in Cancer; p. 25, n. 12 p. 3645- 50, 2018. 


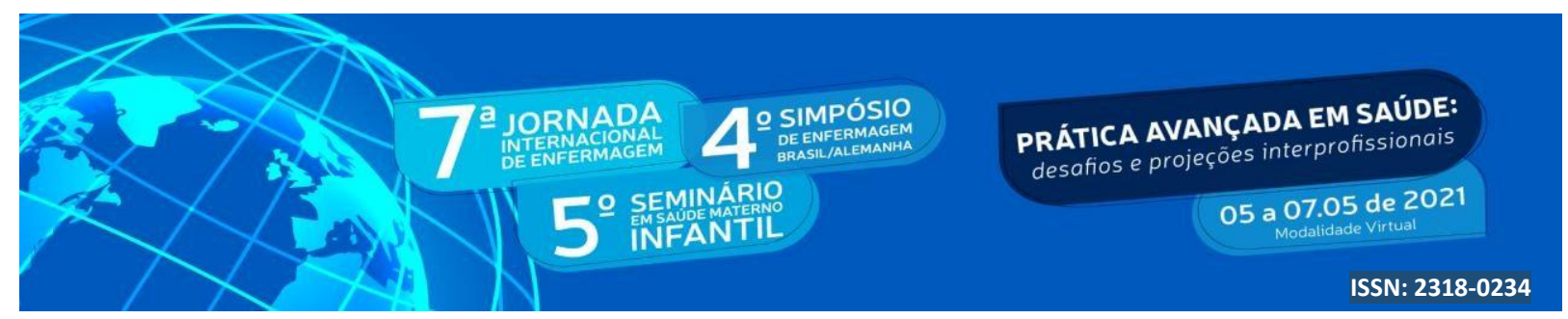

LOPEZ, G.; MAO, J.J.; COHEN, L. Integrative Oncology. Medical Clinics of North America. v. 101, n. 5, p. 977-985, 2017.

MOURA, C.M.; CARVALHO, A.C.; FALCÃO, D.P. Rotulagem de medicamentos fitoterápicos industrializados: está adequada às diretrizes legais? Vig Sanit Debate v. 2, n. 2, p. 60-66, 2014.

QUEST, P.; ROBERTS, K. The Reiki- Manual: a training guide for Reiki students, practitioners, and masters. Hachette Digital, p. 352, London, 2011.

SALIK, A.K. O paciente oncológico e suas relações de encontro. Rev sbph. v. 16, n. 2, p. 89102.

SHALOM, S. I. et al. Impact of a complementary/integrative medicine program on the need for supportive cancer care-related medications. Support Care Cancer. Supportive Care in Cancer; v. 25 , n. 10 p. $3181-90,2017$.

SIEGEL, P.; BARROS, N. F. O que é a Oncologia Integrativa? Cad Saúde Colet. v. 21 n. 3 p. 348-354, 2013.

SILVA, A. N. et al. Política nacional de atuação oncológica: dificuldades e desafios. Braz. J. of Develop. v. 6, n. 9, p. 68354-68368, Curitiba, 2020.

SPADACIO, C.; BARROS, N. F. Uso de medicinas alternativas e complementares por pacientes com câncer: revisão sistemática. Rev Saúde Pública. v. 42, n. 1, p. 158-164, 2008. 